\section{Gold 2003:}

\section{Highlights of this}

International

Conference on the

Science, Technology

and Industrial

\section{Applications of \\ Gold, Vancouver \\ 28 September - \\ 1 October 2003}

This landmark event began on Sunday afternoon with welcoming addresses by Jim Burton, Chief Executive Officer of World Gold Council, and Yvan Jacques, Executive Director, The Canadian Institute of Mining, Metallurgy and Petroleum. The importance of the new gold science currently emerging from research laboratories to the future development of new applications for gold was emphasized. This very well attended first session was then addressed by four keynote speakers who introduced the four principal themes of the conference, ie gold catalysis, chemistry, materials, and nanotechnology.

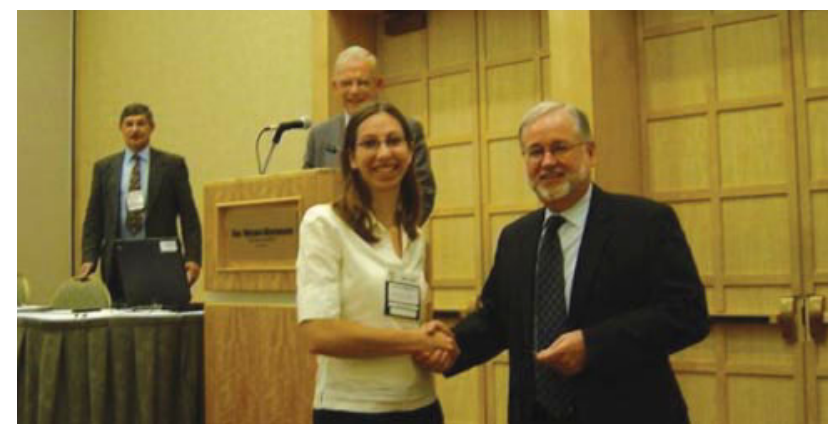

New Directions in Gold Catalysis Graham Hutchings, University of Cardiff, UK, described the increasingly wide use of gold as a heterogeneous catalyst for chemical transformations. It was shown that Au-based catalysts are active and selective in the oxidation of alcohols to aldehydes and acids and in the in situ production of hydrogen peroxide from a mixture of $\mathrm{H}_{2}$ and $\mathrm{O}_{2}$. In particular, $\mathrm{Au}-\mathrm{Pd} / \mathrm{Al}_{2} \mathrm{O}_{3}$ is a very efficient catalyst for the direct manufacture of $\mathrm{H}_{2} \mathrm{O}_{2}$, an oxidant that may, for example, become important in the synthesis of fine chemicals. The influence of gold metal particle size and oxidation state were discussed together with the role of the oxide supports. There has been a marked increase in rate of growth of interest in catalysis by gold in recent years, as illustrated by the rapid increase in numbers of publications.

Fundamental Knowledge in Gold Chemistry: A Sound Basis for Multidisciplinary Development Hubert Schmidbaur, Technical University of Munich, indicated that progress in gold chemistry is possible through a multidisciplinary approach to the subject which allows a deeper understanding of the special position held by gold amongst the metals in the Periodic Table. Theoretical physics and chemistry have become of great value in understanding relations between the structure and intriguing properties of gold and its compounds, and predicting compositions and novel functionality. Theoretical chemistry and relativistic effects have played an important role in understanding the unique character of this element, the relations between the structure and properties of its compounds and, more recently, in the prediction of new derivatives.

The Weird World of the Nanoscale Michael Cortie, University of Technology, Sydney, Australia, indicated the unique potential of gold within this field. Due to plasmon resonance, the colour of gold nanoparticles varies with their size, and gold structures at the bottom end of the nanoscale may, depending on shape and substrate, be semiconductors with a significant band gap. At the nanoscale, gold can be semi-conducting, purple or crystalline. Applications are foreseen in monitoring body fluids, new electronic devices and in decorative effects.

Commercial Aspects of Gold Applications: from Materials Science to Chemical Science Christopher Corti and Richard Holliday, World Gold Council, London, UK, contrasted the traditional uses for metallic gold in industrial applications and jewellery with the new opportunities for gold applications based on the exciting results emerging from current research into gold catalysis, chemistry, and nanotechnology, including fuel cell systems, pollution and emission control, chemical processing and development of new coatings. The availability and price stability of gold provide strong additional incentives to apply gold in new projects being progressed in all four areas represented by the conference. 
There were eleven more keynote lectures, the first three of the following being plenary:

Gold, Relativity and Nanosized Systems Pekka Pyykko, University of Helsinki, Finland, emphasized that relativistic effects have a major influence on the properties of gold and its compounds. Also the aurophilic attraction between $\mathrm{Au}(\mathrm{I})$ ions is enhanced by relativistic effects. $A b$ initio quantum chemistry and isoelectronic thinking can be used to predict new inorganic species: the closed shell icosahedral WAu ${ }_{12}$ has very short W-Au bonds and was synthesized very shortly after this was predicted. This species could be covered with ligands (e.g. Xe, thiolate), and extended to nanostructures and the isoelectronic ions $\mathrm{TaAu}_{12}{ }^{-}$and $\mathrm{ReAu}_{12}{ }^{+}$.

Gold as a Novel Catalyst in the 21st Century: Preparation, Working Mechanism and Applications Masatake Haruta, Research Institute for Green Technology, AIST, Tsukuba, Japan, who was introduced by Geoffrey Bond as "the reason we are all here"!, described the various methods by which active gold catalysts can be prepared. They often exhibit useful catalytic properties below $473 \mathrm{~K}$. They have already been commercially used for deodorizers in Japanese rest rooms and they will find growing applications in indoor air quality control, pollutant emission control, gas masks, production of hydrogen for energy applications such as fuel cells, and innovations in chemical processes. Significant progress was reported in increasing the conversion of propene to propene oxide by optimising the gold catalyst oxide support. The cluster science of gold may also open an exciting area of research showing some magic numbers for dramatic changes in reactivity.

Surface Chemistry of Catalysis by Gold Hajo Freund, Fritz-Haber Institute der Max-Planck-Gesellschaft, Berlin, Germany, reviewed the structure and energetics of gold surfaces prepared on single crystalline material as well as their behaviour towards adsorption and reaction of molecules. These results were then compared with recently gathered information on gold particles down to the nanometer regime. He also discussed the preparation, characterization and reactivity of gold clusters on model oxide supports. Defects on the oxidic support play a crucial role.

\section{Keynote Lectures}

Homogeneous Catalysis by Gold Stephen Hashmi, University of Stuttgart, Germany, provided a general overview of current knowledge on homogeneous catalysis by gold, including addition, condensation and isomerization reactions. Some synthetic reactions are uniquely catalysed by gold, and a few have high turnover numbers. This aspect of gold catalysis is, as yet, at a very early stage of development.
Scope for New Applications for Gold Arising from the Electrocatalytic Behaviour of its Metastable Surface States Declan Burke, University of Cork, Ireland, described the electrocatalytic behaviour of gold in aqueous media and presented a novel view of gold electrode surfaces involving protruding gold atoms functioning as electrocatalytic redox mediators. The advances in the heterogenous catalysis by gold could be accompanied by similar advances in the use of gold in the electrocatalysis area.

Gold Luminescent Materials Alan Balch, University of California, Davis, USA, described two-coordinate gold(I) complexes which display strong photoluminescence and participate in a number of unusual luminescence phenomena. Frequently, the luminescence is associated with the formation of weak but significant aurophilic attractions between gold(I) ions. The role of polymorphism (crystalline isomers) and crystal purity in the observation of luminescence was discussed and their utility as sensors considered. It is possible to modify the optical properties through a systematic perturbation of the nature of the ligands and the steric and electronic effects imposed by the substituents. Many of these compounds can be useful as chemosensors and others have potential applications as sensors for gaseous organic compounds.

Some Recent Developments in Non-Cyanide Gold Plating for Electronics Applications Masuru Kato, Kanto Chemical Company, Saitama, Japan, described work undertaken in collaboration with Yutaka Okinaka. Plated gold as used by the electronics industry can be broadly classified into two categories: soft gold and hard gold. Soft gold is used for circuit metallization and for bonding semiconductor chips, while hard gold is indispensable as the contact material on electrical connectors and electromechanical relays. The traditional baths from which to plate soft gold as well as hard gold contain the cyanide complex, $\left[\mathrm{Au}(\mathrm{CN})_{2}\right]^{-}$, as the source of gold, which liberates free cyanide ions during the plating. The free cyanide is not only highly toxic but also attacks photoresists used to delineate circuit patterns and bonding pads. For these reasons, non-cyanide baths are in use to plate soft gold, whereas hard gold can be plated only from cyanide baths at present. In this presentation, both electrolytic and electroless non-cyanide processes for plating soft gold were described.

Future Applications of Gold in Electronics Tim Ellis, American Competitive Institute, Philadelphia, USA, described how gold-based materials have been the mainstay of the electronics and semiconductor industry since the physics was just a laboratory curiosity. Gold is the workhorse material in wire bond, flip chip and off wafer interconnections due to its corrosion resistance, ability to form metallurgical bonds by soldering or cold welding, and ease of fabrication. Although there is pressure towards miniaturization in the electronics industry, which will reduce the demand for gold, emerging demands in bioelectronics, photonics and power 
requirements in conventional devices represent new opportunities for gold use. A key requirement is for the gold bonding wire industry to become more actively involved in the electronics industry 'Roadmap' initiatives.

The Remarkable Optical Properties of Gold Nanoshells Naomi Halas, Rice University, Houston, USA, said that the unusual colour of ultrasmall gold spheres is due as much to the shape of the particles as to their constituent metal. By varying the shape of gold nanoparticles from a solid sphere to a hollow sphere or 'nanoshell', their colour can be tuned across a large range of the optical spectrum from visible into the infrared. Modern chemical techniques can be used to give precise and systematic control over the size and thickness of the gold nanoshells, allowing the tuning and manipulation of their colour as well as their ability to selectively absorb or scatter light. These new gold colours give rise to a wide range of new applications, most particularly in biomedicine, where the biocompatible qualities of gold combine with their ability to absorb or scatter light in the region of the optical spectrum of highest physiological transparency.

Gold-Based Biosensors for Near-Patient Assessment Bruce Cornell, AMBRI Ltd, Sydney, Australia, described new technology which mimics sensing mechanisms found in biology, but is adapted to be more stable and to be directly compatible with electronic devices for reading and storing data from clinical measurements. The Ambri system works by measuring the changes in the ionic currents induced across a specially designed lipid membrane chemically tethered to a piece of gold-coated plastic. Being chemically tethered to the gold surface results in a very stable membrane whose conduction may easily be measured by simple electronics.

Helical Gold Nanowire Kunio Takayanagi, Tokyo Institute of Technology, Japan, described gold nanowires which take a helical multi-shell structure (HMS) when their diameter becomes less than $2 \mathrm{~nm}$. The HMS has the geometry of multiwall co-axial tubes. The reason for the formation of the gold into metal helicies is not yet known, but it is envisaged that techniques which allow the synthesis of long quantum nanowires with nanometre scale diameters will be useful in the future.

The keynote lectures summarized above will be published in a special edition of Gold Bulletin early next year.

\section{Catalysis}

Altogether, more than 150 lectures and posters were presented of which more than 75 dealt with advances in heterogeneous and homogeneous catalysis by gold. The oral presentations dealing with catalysis were held in two parallel sessions under the names 'Catalytic Gold 1' and 'Catalytic Gold 2'. These names were chosen to demonstrate that the conference was a follow up of the first International
Conference on the progress in gold catalysis - 'Catalytic Gold', held in Cape Town, April 2001, where there had been only 27 presentations on gold catalysis. The titles of the sessions were 'Fundamentals in Cold Catalysis', 'New Routes to Chemicals via Homogeneous Catalysis', 'Applications for Gold Catalysts', 'Fuel Cells and Hydrogen Processing', 'Surface Chemistry and Catalysis', 'Advances in Heterogeneous Catalysis', 'Bimetallic Catalysts', 'Catalyst Preparation and Characterization', 'Gold Catalysts in Chemical Processing', 'New Catalysts', and 'Electro- and Nano-Catalysis'.

In his presentation entitled 'What makes gold an active catalyst?', Harold Kung, Northwestern University, USA, focused on factors relevant to CO oxidation. The nature of the gold active site was also discussed by Mayfair Kung on the basis of recent XANES and XPS studies. Selective hydrogenation of acrolein to allyl alcohol and of crotonaldehyde to crotyl alcohol were discussed by Peter Claus, Darmstadt University of Technology, Germany, and by Catherine Louis (Université Pierre et Marie Curie, Paris, France), respectively.

Michael Bowker, Cardiff University, UK, presented an interesting talk entitled 'Photocatalytic hydrogen production from methanol using Au catalysts'. Au/ $\mathrm{TiO}_{2}$ was found to be more active - on a molar basis - than $\mathrm{Pd} / \mathrm{TiO}_{2}$. Michiel Makkee, of Delft University of Technology, The Netherlands, indicated that disturbed metallic gold is present on gold-titania based catalysts, and this is selective in propene epoxidation.

The prize for the most innovative presentation, a Krugerrand donated by the Rand Refinery in South Africa, was presented by Peter Radcliffe to Michele Rossi (University of Milan, Italy) and his team. It was demonstrated for the first time that naked gold particles have a high initial activity under mild conditions in the oxidation of glucose to gluconic acid, an important food additive. However, a carbon-support is needed to stabilize the small gold particles.

There are also clear signs that homogeneous catalysis by gold in the liquid phase will have a very significant future: Teruyuki Hayashi (AIST, Tsukuba, Japan) described studies in which catalyst systems comprising $\left(\mathrm{Ph}_{3} \mathrm{P}\right) \mathrm{AuCH}_{3}$ and acid cocatalysts were found to be highly efficient for hydration of alkynes to give ketones. Turnover numbers in the range 3,500 to $15,600 \mathrm{~h}^{-1}$ were reported.

Several lectures dealt with multicomponent Au-based catalysts. The addition of another metal (bimetallic catalysts) or various kinds of oxides can result in a more efficient catalyst. For example, Andreea Gluhoi, Leiden University, The Netherlands, showed that in $\mathbf{N H}_{\mathbf{3}}$ oxidation the selectivity to $\mathrm{N}_{2}, \mathrm{~N}_{2} \mathrm{O}$ or $\mathrm{NO}$ can be steered in the desired direction by a proper selection of the oxidic additive.

Donald Cameron, The Interact Consultancy, Reading, UK, presented an overview of the development of fuel cell systems, including use of gold in the generation and 
purification of hydrogen as well as in the fuel cell itself. Potential applications are in stationary generators, cars and buses, industrial vehicles and small portable power suppliers. In this lecture and in the lecture of C.J. Zhong (Binghamton, USA) the huge opportunities for gold catalysts in fuel cell applications were identified. Marcus Kinne, University of UIm, Germany and Chang Kim, University of Michigan USA, both described gold catalysts active in the Water Gas Shift (WGS) for production of hydrogen.

The performance of gold-based catalysts is very sensitive to the preparation method used, the pretreatment of the catalyst and to the experimental conditions. It is helpful, therefore that the World Gold Council has taken the initiative to supply a number of reference gold catalysts prepared by Süd Chemie for AIST in Japan under the direction of Drs Masatake Haruta and Susumu Tsubota, and by Professor Michele Rossi in Milan. Dr Tsubota discussed the characterization and catalytic activity of the oxide-supported reference catalysts in the oxidation of $\mathrm{CO}$ and $\mathrm{H}_{2}$.

During the conference and, in particular, during the discussions in the Open Forum (see below), the different views concerning the effect of the particle size, the possible roles of ionic gold and metallic gold, and the nature of the active site/species were discussed in detail. The poster session was also lively and interactive with over 25 posters covering different areas from industrial catalysis to surface science studies.

We learned a lot about the intrinsic properties of gold. C.P.Vinod (Eindhoven University, the Netherlands) showed that low-coordinated Au atoms are able to decompose NO at low temperatures. Norbert Kruse (Université Libre de Bruxelles, Belgium) presented in his lecture images with atomic resolution for $\mathrm{CO}$ oxidation on a model gold catalyst: low-coordinated gold atoms are the active centres.

Veronique Pitchon (CNRS-ECPM, Strasbourg, France) described the preparation of strongly anchored gold catalysts, which are choride free and stable at $600^{\circ} \mathrm{C}$ : these are reactive with $\mathrm{C}$, to $\mathrm{C}_{3}$ hydrocarbons under conditions appropriate for automotive exhaust cleaning.

It was shown in several lectures that gold-based systems are active and selective catalysts for a variety of important

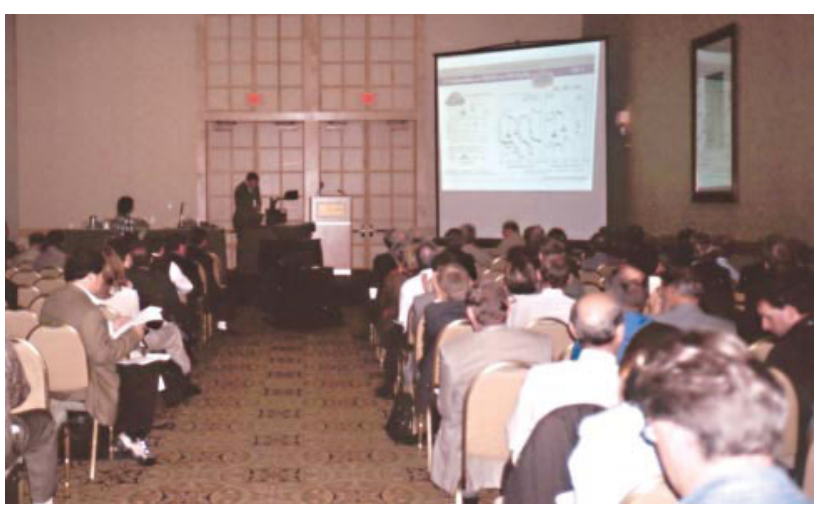

application reactions: e.g. selective oxidation and other hydrogenation of hydrocarbons, production of $\mathrm{H}_{2} \mathrm{O}_{2}$, selective oxidation of $\mathrm{NH}_{3}$ to $\mathrm{N}_{2} \mathrm{O}$, and reactions relevant for pollution control, including indoor air purification and even the emission of mercury (via its oxidation) from coal-fired power stations.

The presentations in the Catalysis section therefore demonstrated that there is likely to be a golden future for gold catalysis in terms of a wide range of new commercial applications.

\section{$4 \quad$ Chemistry}

There were 19 oral and 8 poster presentations in the Chemistry section. The topics were focused not only on the most important aspects of the chemistry of this precious metal, but also in the potential new commercial applications of its compounds. The chemistry programme was structured in six sessions: 'Theoretical Chemistry and Reaction Mechanisms', 'Photoluminescent Compounds for Optronics', 'Pharmaceutical Applications', 'Chemistry Applications' and two on 'New Gold Chemistry'.

Peter Schwerdfeger, Auckland, New Zealand, in his talk entitled 'Relativistic effects in the chemistry of gold - from small molecules to nanostructured materials' provided a useful practical sequel to the keynote lecture given earlier by Professor Pyykko. Some polynuclear systems have Iuminescent properties and these were analysed in a number of talks: Vivian Yam, Hong Kong University, China, in 'Design of luminescent gold complexes as functional materials and chemosensors' and Conchita Gimeno, University of Zaragoza in "polynuclear chalcogenide-centered complexes with metallophilic interactions' described the use of chalcogenide complexes; and dinuclear gold phosphine complexes bearing ethynylfunctionalized calix[4] crown moieties, that change the electronic absorption spectra after reaction with alkali metals were also described by Professor Yam. Octafluoronaphthalene and pyrazolate luminescent derivatives were described by John Fackler, Texas A\&M University, USA, in his talk entitled 'Gold(I) as a nucleophile - a base'. The neutral $\pi$-acids $C_{6} F_{6}$ TCNQ form $\pi$ acid, $\pi$-base products with stacked trinuclear Au(I) compounds: the structure of the octafluoronaphthalene acid-base product displays a bright yellow photoluminescent emission. Antonio Laguna, University of Zaragoza, Spain, described the strongly luminescent heteropolynuclear complexes obtained by reaction between basic gold precursors and acid silver or thallium derivatives which are very good sensors for volatile organic compounds: in these cases the change of colour and luminescent properties are due to interactions of the ligands with the silver or thallium centres.

Daniel Leznoff, Simon Fraser University, Vancouver, in a talk 
entitled 'Coordination polymers with cyanoaurate building blocks: structure, physical properties and applications' described other types of sensors and molecular storage materials, based on cyanoaurate derivatives, that show large colour changes by absorption of a variety of ligands in the open spaces of the molecular structures. The prize for the best student poster was presented by Jay Taylor, President and CEO of Placer Dome, Canada, to Julie LeFebvre from the Simon Fraser group for her presentation entitled ' $\left[\mathrm{Au}(\mathrm{CN})_{2}\right]$ based polymers for materials applications' which described the straightforward syntheses of the inorganic polymers which are likely to find applications as solvent and gas sensors.

The uses of different gold(I) or gold(III) compounds as chemical sensitizers of silver halides or phosphorescent gold(I) derivatives as dopants in organic light emitting diodes (OLEDs) in imaging technology was discussed by Henry Gysling, Eastman Kodak Company, Rochester, NY, USA. Many such compounds exhibit phosphorescence, with emissions throughout the visible spectral region, with lifetimes of the order of microseconds and emission quantum efficiencies of $0.1-0.3$.

The use of gold compounds in medicine was discussed in the Pharmaceutical Applications Section. Since the introduction of auranofin (1985) in the treatment of rheumatoid arthritis there has been no new clinically approved gold drugs, but a broad range of gold compounds has been evaluated and antitumour activities found. Sue Berners-Price, University of Western Australia, in her talk entitled 'Gold-phosphine and gold-carbene complexes as potential mitochondrial targeting anti-tumour agents' described the latest results in the design of gold drugs with optimal lipophilic-hydrophilic character, i.e. with hydrophilic pyridylphosphine or heterocyclic carbene as ligands, able to target mitohcondrial cell-death pathways. Edward Tiekink, National University of Singapore highlighted the anti-tumour potential of gold compounds shown during studies with phosphinegold (I) thiolates, especially when the thiolate ligand is derived from a sulfur-containing drug or metabolite that exhibit high cytotoxicity in cisplatin-resistant cell lines. Simon Fricker of Anormed, Canada discussed the potential activity of gold(III) derivatives with 2-[(dimethylamino)methyl]phenyl as ligand. Some of them show activity against cysteine proteases that have been proposed to play a role in tumour metastasis and progression, or against a panel of human tumour cell lines. Finally, Frank Shaw analysed the biochemical and medical aspects of the dicyanoaurate(I) anion: its ability to carry gold into cells, the behaviour as a metabolite responsible for the antiarthritic activity of gold, or the possibility to be oxidised to tetracyanoaurate(III) by hypochlorite (an oxidant released by immune cells).

Richard Puddephatt, University of Western Ontario, Canada, gave a talk entitled 'A gold standard for molecular materials: gold rings, catenanes and polymers' and Maria Cinellu, University of Sassari, Italy, one on 'Reactions of gold(III) oxo complexes with $\mathrm{PPh}_{3}$ and alkenes' described other significant advances in gold chemistry, with potential applications as molecular materials or in catalysis. Professor Puddephatt analysed the selective formation of gold rings or polymers by using bridging ligands and how to manipulate the ring cavity size of the macrocycle to promote interpenetration to form catenanes or how to induce helicity in polymers. Dr Cinellu's paper described some nice routes for the synthesis of oxo and hydroxo gold derivatives, a type of derivative not previously widely represented in gold chemistry.

The Chemistry Section of the Gold 2003 Conference not only showed how much research in this field is growing, but also guaranteed an important role for this element in some emerging applications. Increasing the knowledge of gold chemistry will have useful spin-off into new approaches in homogeneous and heterogeneous catalysis as well as nanotechnology.

\section{$5 \quad$ Materials}

Five Materials-related sessions were held during the GOLD 2003 conference under the titles 'Innovations in Electroplating', 'Gold in Medical and Dental Applications', 'New Electronic Materials' (2 sessions) and 'Advanced Gold Alloys', resulting in a total of 16 presentations in all. A number of posters also focused on gold materials related topics.

Gold electroplating is used extensively within the electronics industry for achieving the performance characteristics offered by gold at an affordable cost. Andreas Zielonka from FEM, Germany, described how new electrodeposited gold-nickel/cobalt multilayer systems could be produced for contacts and connectors. Enhanced and novel properties of these materials could find application, for example, in automotive connector applications.

Although the use of gold in dentistry and other medical uses dates back thousands of years, new technology is expanding the scope of applications for gold in these fields. Presentations in this session described the growing use of electroforming for dental prosthesis production and its emerging innovative use as a cost effective way of manufacturing medical implants, specifically myringotomy tubes for drainage of the tympanic cavity (Heidi Fauser, FEM, Germany). Paul Cascone from the Argen Corporation, USA described the development of a new high gold content casting alloy with small additions of indium and zinc. This alloy was reported to offer improved aesthetic qualities over existing alloys, with the required mechanical properties for some porcelain-fused-to-metal restorations.

The two sessions devoted to emerging applications in the electronics area described new gold materials for improved bonding wires and solder alloys. New gold alloy metal-metal 
matrix composites were described, with potential application in high conductivity and high strength bonding wires (Kageeporn Wongpreedee, lowa State University, USA). Masayuki Ishikawa, Mitsubishi Materials Corporation, Japan described the application of gold-tin solder pastes for fine pitch devices in radio frequency and optoelectronic device packaging. These can be applied flexibly and have achieved void reduction by optimising powder size, flux composition and reflow condition. A major new potential use for gold was identified by Eamonn Maher, 3C's, UK, who described the need for metallic protection layers on new low temperature superconducting tapes for use in power distribution and superconducting magnets. Based on the complex requirements for high thermal and electrical conductivity, high specific heat capacity, and resistance to oxidation, gold is envisaged as a likely candidate material for the protection layers.

Improvements in the chemical, physical and mechanical properties of a material offer the opportunity to ultimately expand and diversify its industrial applications. The final materials related session was therefore devoted to the latest research on improved properties of advanced gold alloys. Joerg Fischer Buehner, FEM, Germany, described how the age hardening behaviour of 22ct and 990/995 Au alloyed with Co or $\mathrm{Ni}$ could be significantly influenced by additions of Sb. The alloys developed from his work could be exploited in electronics applications, alongside jewellery and decorative uses. Liz Glaner, Mintek, South Africa, presented the results of an investigation into the influence of additions of gold to Fe-Cr-Al ('Fecraloy') alloys used in industry as substrates for high performance metallic catalysts. 5wt\% additions of Au to 'Fecraloy' were found to be beneficial to the oxidation resistance of the alloy at high temperatures.

The lively poster session allowed those delegates with a primary interest in materials related topics to interact with delegates from the other three technical themes. Materials related posters included those prepared by Olaf Andersen from the Fraunhofer Institute for Manufacturing and Advanced Materials, Germany who had carried out work on the melt extraction of gold fibres and preparation of porous gold fibre structures. On-going work at his centre was focused on developing applications utilising the functional properties of the sintered fibre structures. Felix Levin, Unifine Works Ltd, Israel, described a promising approach for a cyanide free gold immersion method for producing a wide range of gold coating thicknesses.

In conclusion, the high quality of the presentations in the Materials sessions fully contributed to the overall success of the conference and most importantly highlighted some very promising new technologies that had the potential to lead to the increased application of gold in industry.

\section{Nanotechnology}

The nanotechnology sessions were entitled 'Manufacture of Nanoparticles', 'Nanoparticle Composites', 'Advances in Nanotechnology', 'Future Horizons in Nanotechnology' and 'Nanotechnology Applications'. In addition there was also a multidisciplinary session on 'Cold Extraction' which contained nanotechnology papers on mineral nanotechnology by Hugh Abercrombie of Birch Mountain Resources, Canada, and gold phytomining by Christopher Anderson from Massey University, New Zealand.

Michael Cortie, University of Technology, Sydney, talked about potential applications for mesoporous gold. It has the unique property of being both metallic and highly resistant to oxidation. This material is relevant to potential applications in catalysis, biosensors, optical coatings, in analytical chemistry and as a basis for a new kind of ultra-capacitor. Christina Graf, Utrecht University, The Netherlands talked about the enhancement of fluorescence by surface plasmons in gold dielectric core-shell colloids for photonic applications. By varying the thickness of the gold layer and the radius of the shell it is possible to adjust the plasmon resonance of the gold shell particles over the whole visible and infrared region of the spectrum. The use of gold CDs as biochemical sensors was described by Hogan Yu, Simon Fraser University, Vancouver: after removing the protective polymer film with nitric acid, species in body fluids containing -SH groups would react with the gold to form self-assembled monolayers on the surface. It was also confirmed that these substrates are suitable not only for surface wetting measurements, but also surface electrochemical studies.

Ayako Iwakoshi of Nippon Paint, Japan described highly concentrated pastes of gold nanoparticles produced using a special kind of protective polymer and a mild reductant. The solid part of the paste consists of up to $85 \mathrm{wt} \%$ of gold nanoparticles $10-30 \mathrm{~nm}$ in diameter, and a residual protective layer. The application fields foreseen include electronics, optics, catalysts, colourants and decorative surface metal coatings. Masami Nakamoto from Osaka Municipal Technical Research Institute, Japan, described production of useful gold nanoparticles by controlled thermolysis of gold thiolate complexes: the rate of growth of the gold nuclei varied according to heating temperature and reaction time.

Peter Bishop, Johnson Matthey Technology Centre, Reading, UK, described the use of gold inks for decorative applications: these inks contain soluble nanoparticle precursors that thermally decompose to form bright gold films. Preparation of thiol-stabilized gold nanoparticles has been achieved using the monolayer protected cluster methods first described by Brust et al (University of Liverpool, UK) and stabilizing ligands employed have been aromatic thiols, monosubstituted with branched alkyl moieties. 
Toshiharu Teranishi, AIST, Japan, has developed a novel synthetic procedure to precisely control the particle size of alkanethiol-protected gold nanoparticles in the range from 1.5 to $9.7 \mathrm{~nm}$ by the heat treatment of the small gold nanoparticles in the solid state. The absorption and nonlinear optical properties of these materials were investigated and the fabrication of gold nanoparticle superlattices is envisaged having useful optical and electronic properties. The development of methodology to order the size and shape of gold nanoparticles in desired patterns will be the key technology for developing nanodevices in the future.

Amongst other interesting presentations in this section was one by Jillian Buriak, National Research Council, Edmonton, Canada, who described patterning of gold nanoparticles on semiconductor surfaces through electroless deposition. It has recently been demonstrated that the technique is compatible with photolithography, microcontact printing and dip-pen nanolithography.

We can therefore conclude that although still very young, the emerging field of gold nanotechnology can be envisaged as having wide applications in new device technology and decorative applications and we must also bear in mind that the rapidly developing area of gold catalysis has a significant input from gold nanotechnology.

\section{Open Forum: A Feisty Monday Evening in Vancouver BC}

The unseasonably fine weather and the waterfront location of the 'Open Forum Discussion' on the Monday evening had given the organizers of this event a furrowed brow or two. However, it appeared that the crisp late summer waterfront air was no match for a potential good barney, and the delegates to GOLD 2003 attended the informal 'Open Forum' in large numbers. The intention of the organizers was to stimulate a lively debate in respect of gold catalysis and gold ligand chemistry, and in this respect they were not disappointed.

The event was moderated by David Thompson (WGC, UK) who introduced the topic 'Why is nanoparticulate gold so special' and asked about the mechanism of gold catalysis and the durability and poison resistance of gold catalysts. The discussion was then set in motion by a few opening speakers, each restricted to two minutes each. Professor Michael Cortie of University of Technology, Sydney, kicked off by first declaring that he was merely an interested outside observer of matters catalytic (and therefore presumably a neutral party), but that in his view the field was so far characterized by a frustrating irreproducibility and that the theories advanced to date appeared to lack broadly predictive capability. Furthermore, due to experimental scatter, it was unlikely that an analysis of rate versus particle size was likely to be much use to differentiate the effects due to perimeter activity, surface defects or electronic state.

The next speaker, Mayfair Kung of Northwestern University, USA, advanced the view that a unique characteristic of gold catalysts was that they required adsorbed water (to displace $\mathrm{CO}_{3}{ }^{2-}$, a poison), and that in her experience these catalysts performed very poorly in dry conditions. This set the stage for the next speaker, Professor Ben Nieuwenhuys of Leiden, The Netherlands, who put up an overhead showing a number of possible mechanisms and sites for catalysis by gold. However, he seemed to favour the effect being due to the presence of special, active sites, presumably of low co-ordination number, with the role of the support being very much a secondary factor.

Next up to the podium was Dr Masatake Haruta of Tsukuba in Japan. He proposed that the effect was 'multi-functional'. However, its essential ingredients in his opinion were that a metallic nanoparticle of gold needed to be present, and that this particle had to be attached to an oxide support. The oxygen would come via the support, the reductant (e.g. CO) would adsorb onto the gold, and that the reaction would take place along the perimeter of the $\mathrm{Au} / \mathrm{M}_{\mathrm{x}} \mathrm{O}_{\mathrm{y}}$ interface. He further stated that the perimeter gold would have to be in a partially oxidized state, but that in his view water was not necessary if the gold was supported on reducible oxides.

The forum then mutated into a general discussion, with Michael Cortie advancing the claim that at least some studies existed which showed that samples lacking $\mathrm{Au}^{0}$ could be active. The co-author of one such study cited by Cortie, Graham Hutchings of Cardiff, UK, disputed this interpretation and reminded the audience that the in situ oxidation state of the gold could hardly be deduced with certainty from an ex situ characterization. However, Maria Stephanopoulos, Tufts University, USA, author of a recent paper published in Science on this subject stood up and confirmed that they believed that, at least in some cases, such as the WCS, highly active behaviour could be shown in the absence of $\mathbf{A u}^{0}$. On the question of reproducibility raised by Cortie, Hutchings noted he considered the results of his group to be adequately reproducible, but conceded that consistency between labs was still unsatisfactory.

The discussion then returned to the issue of where the oxygen came from that oxidised CO, for example, Professor Geoffrey Bond, reminded the meeting that isotope studies carried out by Professor Iwasawa, Tokyo, Japan, seemed to indicate that it was only gas-phase (i.e. not lattice) oxygen that did the job. However Masatake Haruta disputed this and noted that their measurements had found that the oxygen had indeed come from the $\mathrm{M}_{\mathrm{x}} \mathrm{O}_{\mathrm{y}}$ support. This solicited a comment from Hajo Freund of the Max Planck Institute, Berlin, who stated that, in his experience with $\mathrm{MgO}$ at least, only gas phase oxygen took part. While the meeting pondered these views, Harold Kung of Northwestern 
University, USA, commented that in his experience the oxygen actually came from water adsorbed on the oxide support. Furthermore, he took issue with the hypothesis that there might be several kinds of active site in these catalysts.

Next even the necessity for an oxide support came under scrutiny. Declan Burke noted that electrochemically cycled gold contained a metastable surface which was readily oxidized, and catalytically active, with the catalysis possibly involving a redox cycle between $\mathrm{Au}^{+}$and $\mathrm{Au}^{3+}$. An active site of this nature did not depend on the presence of an $\mathrm{M}_{\mathrm{x}} \mathrm{O}_{\mathrm{y}}$ support either. The following day (see above) Professor Michele Rossi of Milan set the cat amongst the pigeons by presenting direct evidence of his own for oxidation of glucose under mild conditions in a reaction involving only $\mathrm{Au}^{0}$ particles.

The moderator, David Thompson, then steered the Forum in the direction of a new topic 'Which ligands would rejuvenate gold chemistry'. Gold chemistry is as yet underdeveloped compared with that of the Platinum Group Metals, and the injection of new ideas would lead to new types of compound, such as gold hydrides, suitable for homogeneous catalysis and other new applications. Pekka Pyykko of Helsinki, Finland showed that gold really is unique, at least in respect of the relativistic effects imposed by its nucleus. Gas phase chemistry, while not bountiful, had established a reliable ranking of possible ligand species, with the Au-Xe bond the weakest and the Au- $\mathrm{PH}_{3}$ the strongest. However, the fact that gold had an interaction with Xe at all was an outcome of the relativistic effect. As far as new ligands are concerned, he speculated that $\mathrm{GeX}_{3}$ and $\mathrm{AsF}_{3}$ might have potential, but that their malodorous nature was a significant disincentive.

John Fackler, of Texas A\&M University, USA, mentioned that a variety of interesting nitrogen-containing ligands had recently been uncovered, that the results seem surprising and perhaps worthy of closer examination. Professor Antonio Laguna of Zaragoza, Spain, agreed that gold has a unique chemistry, and for example both the $5 \mathrm{~d}$ and the $6 \mathrm{~s}$ shells were available for bonding. The gold-gold interaction was so strong that it could overcome Coulombic repulsions. This set the stage, he said, for the synthesis of a variety of interesting layered and stacked organometallic molecules.

Vivian Yam from Hong Kong brought the discussion back to ligand chemistry by noting that the possibility of aurophilic effects made the behaviour pretty interesting, even without new ligands. However, it would certainly be possible to substitute selenium for sulfur. It would be possible to control the luminescence and phosphoresence by such substitutions, and to bring these optical effects into the near-infrared.

David Thompson asked the meeting whether there were any other thoughts on new ligands. Alan Balch of University of California, Davis, suggested a different approach. The 'same old ligands' could be used but he suggested that the environment outside of the first coordination shell be targeted as a means of influencing the interior aurophilic bonds. Professor Hubert Schmidbaur, Munich commented that the issue of 'new ligands' was perhaps not that important. The important message he said was that it was possible to manipulate gold through its ligand chemistry. However, he reflected that perhaps some renewed attention to oxygen and nitrogen bonding might nevertheless be worthwhile, since the focus seems to have been on sulfur and phosphorus for too long. Peter Bishop, Johnson Matthey Technology Centre, Reading, UK, said that greater emphasis on gold solution chemistry rather than investigations related to the solid state should lead to increasingly innovative approaches: oxygen, nitrogen and carbene ligands could be useful in this connection.

John Fackler reminded the meeting that there were many interesting and unresolved issues to explore still, for example, the nature of the unstable structure in the explosive 'fulminating gold' precipitated with $\mathrm{NH}_{4} \mathrm{OH}$ was still unknown. In a similar spirit Geoffrey Bond noted that fluoride interactions with gold should not be neglected. David Thompson reminded the meeting that even the interaction with sulfur was not entirely understood, and noted that, surprisingly sulfur did not inevitably poison gold catalyst surfaces, as the work on dehydrosulfurization reactions recently carried out by Anna Maria Venezia (Palermo) had shown.

At this point this evening gathering was brought to a close, not because of any feeling that the possibilities for gold had been exhausted, but rather because a fine warm late summer evening on the Vancouver waterfront cannot be neglected - even for gold.

One of the highlights of the conference was the Tuesday evening banquet at which Peter Radcliffe, Managing Director, Rand Refinery, South Africa, delivered an inspiring after-dinner speech describing the importance of developing new applications for gold for the gold mining industry and the community as a whole, and indicating his belief that the advances described at GOLD 2003 did indeed make this a landmark event in this field. Gold is not merely element 79 but also due to its rarity, beauty and nobility it has been a major factor in shaping the course of history and this role will continue in the future, paticularly in a new technological sense. There was throughout the entire conference an atmosphere of excitement engendered by the many papers on new advances in the four theme areas, and attendance was impressively high at all the sessions. The enthusiastic gathering closed with a lunch on Wednesday addressed by Jay Taylor, President and CEO of Placer Dome, Canada, and the delegates left with very positive intentions to pursue their interests in gold with even greater enthusiasm!

Michael Cortie, Richard Holliday, Antonio Laguna, Ben Nieuwenhuys and David Thompson

Technical Committee Chairmen and Technical

Programme Coordinator GOLD 2003 